\title{
Temperature and magnetization dependent band-gap renormalization and optical many-body effects in diluted magnetic semiconductors
}

\author{
Ying Zhang and S. Das Sarma \\ Condensed Matter Theory Center, Department of Physics, \\ University of Maryland, College Park, MD 20742-4111
}

(Dated: November 5, 2018)

\begin{abstract}
We calculate the Coulomb interaction induced density, temperature and magnetization dependent many-body band-gap renormalization in a typical diluted magnetic semiconductor $\mathrm{Ga}_{1-x} \mathrm{Mn}_{x} \mathrm{As}$ in the optimally-doped metallic regime as a function of carrier density and temperature. We find a large $(\sim 0.1 \mathrm{eV})$ band gap renormalization which is enhanced by the ferromagnetic transition. We also calculate the impurity scattering effect on the gap narrowing. We suggest that the temperature, magnetization, and density dependent band gap renormalization could be used as an experimental probe to determine the valence band or the impurity band nature of carrier ferromagnetism.

PACS numbers: 78.30.Fs;75.10.-b;75.10.Lp;71.45.Gm
\end{abstract}

\section{INTRODUCTION}

A central open question ${ }^{1}$ in the physics of carriermediated ferromagnetism in diluted magnetic semiconductors (DMS) is the nature of the semiconductor carriers mediating the ferromagnetic interaction, in particular, whether the carriers (i.e. the holes in the wellstudied $\mathrm{Ga}_{1-x} \mathrm{Mn}_{x}$ As system) are itinerant band carriers (i.e. holes in the GaAs valence band for $\mathrm{Ga}_{1-x} \mathrm{Mn}_{x} \mathrm{As}$ ) residing in the (valence) band of the parent semiconductor or impurity band carriers residing in a narrow impurity band within the band gap of the semiconductor. This issue is of fundamental significance ${ }^{2}$ since the valence band $\mathrm{3}^{3}$ or impurity band ${ }^{4}$ competing pictures respectively imply competing RKKY-Zener ${ }^{5}$ or double exchange ${ }^{\underline{6}}$ mechanisms for DMS ferromagnetism. In spite of intense experimental and theoretical activity in $\mathrm{Ga}_{1-x} \mathrm{Mn}_{x}$ As over the last ten years, the key issue of valence band versus impurity band carriers mediating the DMS ferromagnetism has remained controversial even in the optimally doped, $(x \sim 0.05)$ nominally metallic, high$T_{c}\left(T_{c} \sim 150-200 K\right.$, the ferromagnetic transition temperature or the Curie temperature) $\mathrm{Ga}_{1-x} \mathrm{Mn}_{x}$ As material. For example, ab initio first principles band structure calculations ${ }^{7}$ typically indicate a strong-coupling narrow impurity band behavior whereas the extensively used phenomenological mean-field description, parameterized by a single effective impurity moment-carrier spin ("pd") exchange interaction ${ }^{1.3 .4 .8}$, leads to reasonable quantitative agreement with experimental results in the metallic $(x \sim 0.05) \mathrm{Ga}_{1-x} \mathrm{Mn}_{x}$ As requiring a relatively weak exchange coupling (and therefore, a weak perturbation of the GaAs valence band) between the Mn moments and the valence band holes. Similarly, optical absorption spectroscopic data in GaMnAs were first interpreted 9 using an impurity band theoretic description ${ }^{10}$, but later it was shown 11 that the same data could also be explained as arising from the valence band picture. Given the great complexity of many competing interaction and disorder effects in the DMS Hamiltonian, it is increasingly clear that this important question cannot be settled purely by theoretical means, and an unambiguous experimental test is warranted.

In this paper we propose an experimental (optical) measurement of the carrier-induced many-body band gap renormalization (BGR) in $\mathrm{Ga}_{1-x} \mathrm{Mn}_{x}$ As for the definitive resolution of this controversy; in particular, we establish theoretically that the BGR in $\mathrm{Ga}_{1-x} \mathrm{Mn}_{x}$ As should be $e x$ tremely large $(\sim 0.1 \mathrm{eV})$ and a strong function of hole density if the carriers are indeed GaAs valence band holes, allowing for a clear distinction between the (valence band) RKKY-Zener and the (impurity band) double exchange mechanisms for DMS ferromagnetism. In addition, we calculate the temperature (as well as density) and hole spin-polarization dependent BGR in $\mathrm{Ga}_{1-x} \mathrm{Mn}_{x} \mathrm{As}$, finding a strong quantitative dependence of the BGR on the magnetic properties - in particular, the calculated BGR depends strongly on whether the system is ferromagnetic or paramagnetic with the ferromagnetic $\mathrm{Ga}_{1-x} \mathrm{Mn}_{x} \mathrm{As}$ typically having a factor of 1.5 to 2 higher BGR than the corresponding paramagnetic system at the same density (and temperature). Our predicted density, temperature, and spin-polarization (i.e. ferromagnetic being fully spin-polarized and paramagnetic being spinunpolarized) dependence of BGR in $\mathrm{Ga}_{1-x} \mathrm{Mn}_{x} \mathrm{As}$ (provided the carriers are the usual GaAs valence band holes) should enable a clear distinction between the valence band and the impurity band picture of DMS ferromagnetism through a careful analysis of experimental optical absorption data 12 . We want to emphasize that our work is not only about studying BGR in the $\mathrm{Ga}_{1-x} \mathrm{Mn}_{x} \mathrm{As}$ system. Our main purpose is to propose a technique for distinguishing whether the GaMnAs carriers are in the valence band or the impurity band, which is a subject of considerable importance and controversy in the DMS community as exemplified by many published works on the topic. On the other hand, to our knowledge the temperature and magnetization dependence of the BGR are never studied before, and they are crucial to the experimental observation of BGR.

Free carriers (e.g. holes in $\mathrm{Ga}_{1-x} \mathrm{Mn}_{x} \mathrm{As}$ ) affect the band gap of semiconductors in two essential ways. First, 
there is the trivial single-particle effect of band filling (sometimes called the Moss-Burstein shift) arising from a finite Fermi level $E_{F}\left(\propto n^{2 / 3}\right.$, where $n$ is the free carrier density) in the valence or the conduction band of the semiconductor as the band is filled with a finite carrier density. The band filling effect obviously increases the apparent band gap by an amount $E_{F}$ which should be subtracted out from the measured band gap energy. In our results presented in this paper we subtract out the trivial band filling Moss-Burstein shift in order to avoid any confusion. The second effect, which is the main subject of this work, is the self-energy correction of the semiconductor band edge due to the many-body exchangecorrelation effect arising from finite carrier density. The band gap renormalization 13.14 is a true many-body effect arising from the hole-hole Coulomb interaction in the $\mathrm{Ga}_{1-x} \mathrm{Mn}_{x}$ As valence band, with the intrinsic (i.e. the zero carrier density limit) band gap being increasingly reduced (i.e. renormalized) by the carrier-induced selfenergy correction as the carrier density increases. This density dependent many-body self-energy correction induced reduction or renormalization of the semiconductor band gap, the BGR, in $\mathrm{Ga}_{1-x} \mathrm{Mn}_{x}$ As is the subject matter of our work.

The rest of the paper is structured as following: In Section [I] we present the formalism of our BGR calculation as a function of temperature, carrier density and spin degeneracy. In Section III we describe the result of BGR for an optimally-doped metallic $\mathrm{Ga}_{1-x} \mathrm{Mn}_{x}$ As DMS system. In Section \V we discuss the implication of our BGR results how they might serve as a probe to determine the valence band or impurity band nature of carrier ferromagnetism, which is the purpose of this paper. Especially, we discuss the effect of impurity scattering on the $\mathrm{Ga}_{1-x} \mathrm{Mn}_{x}$ As optical properties, and why in spite of this complication, the many-body BGR effect can still be experimentally observed.

\section{FORMALISM}

(a)

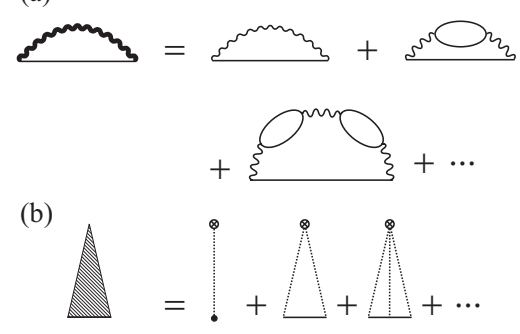

FIG. 1: Feynman diagrams for calculating the quasihole selfenergy. The solid line denotes the hole propagator. (a) oneloop quasihole self-energy due to Coulomb interaction, with the wiggly line denoting long-range Coulomb interaction; (b) quasihole self-energy due to impurity scattering in the singlesite approximation, with the dashed line denoting short-range impurity interaction.
We calculate the BGR in the single-loop (Fig. 10 selfenergy theory which is the leading-order approximation in the dynamically screened Coulomb interaction. This approximation, often referred to as the "GW approximation" 15 in the semiconductor literature $\frac{16}{}$, is known to be exact in the high carrier density limit which happens to be the situation of interest to us since $\mathrm{Ga}_{1-x} \mathrm{Mn}_{x} \mathrm{As}$ is an extremely heavily hole-doped system with the hole density $n \approx 10^{20} \mathrm{~cm}^{-3}$. In $\mathrm{Ga}_{1-x} \mathrm{Mn}_{x}$ As the free carriers are holes, so we only consider the self-energy correction to the band gap arising from the quasihole self-energy correction to the valence band edge within the $\mathrm{GW}$-approximation (Fig. 1), obtaining for the finite-temperature Matsubara self-energy ( $\hbar=k_{B}=1$ throughout):

$$
\begin{array}{r}
\Sigma\left(\mathbf{k}, i \nu_{l}\right)=-\int \frac{d^{3} q}{(2 \pi)^{3}} T \sum_{\omega_{n}} \frac{v_{q}}{\epsilon\left(\mathbf{q}, i \omega_{n}\right)} \\
\times \frac{1}{i \nu_{l}+i \omega_{n}-\xi_{\mathbf{q}-\mathbf{k}}},
\end{array}
$$

where $v_{q}=4 \pi e^{2} / \kappa q^{2}$ is the $3 \mathrm{D}$ bare Coulomb interaction with $\kappa$ the lattice dielectric constant, $i \nu_{l}=i(2 l+1) \pi T$ and $i \omega_{n}=i 2 n \pi T$ are the usual fermion/boson odd/even Matsubara frequencies ( $l, n$ integers), $\xi_{\mathbf{k}}=k^{2} /(2 m)-\mu$ with $\mu$ the chemical potential and $m$ the bare band mass, and $\epsilon\left(\mathbf{k}, i \omega_{n}\right)$ is the dynamical dielectric function, given by the infinite sum of the polarization bubble diagrams:

$$
\epsilon\left(\mathbf{k}, i \omega_{n}\right)=1-v_{q} \Pi\left(\mathbf{k}, i \omega_{n}\right),
$$

with $\Pi\left(\mathbf{k}, i \omega_{n}\right)$ the 3D hole polarizability:

$$
\Pi\left(\mathbf{k}, i \omega_{n}\right)=g \int \frac{d^{2} q}{(2 \pi)^{2}} \frac{n_{F}\left(\xi_{\mathbf{q}}\right)-n_{F}\left(\xi_{\mathbf{q}-\mathbf{k}}\right)}{\xi_{\mathbf{q}}-\xi_{\mathbf{q}-\mathbf{k}}+i \omega_{n}},
$$

where $n_{F}(x)=1 /\left(e^{x / T}+1\right)$ is the Fermi distribution function and $g$ the hole spin degeneracy factor.

In $\mathrm{Ga}_{1-x} \mathrm{Mn}_{x}$ As the valence band structure is complicated with spin-split light and heavy holes both being important. Fortunately, our calculated results for BGR do not depend strongly on the carrier effective mass, and change little if the band mass is changed between $0.3-0.6 m_{e}$. We choose, consistent with experiment, $m=0.5 m_{e}$ as the average band mass in all of our calculations. The dielectric constant $\kappa$ in the system is 10.9 . The spin degeneracy $g$ varies from 1 to 2 according to the magnetization of the DMS system. $(g=2(1)$ for the paramagnetic (ferromagnetic) case.) Spin-orbit coupling effects, which are known to be important for GaAs valence band, are neglected in our theory - spin-orbit coupling is expected to lead to small quantitative corrections of our calculated BGR.

To calculate the retarded self-energy $\Sigma\left(\mathbf{k}, i \nu_{l} \rightarrow \omega+\right.$ $\left.i 0^{+}\right) \equiv \Sigma(\mathbf{k}, \omega)$, we perform a certain contour distortion to transfer the real frequency integration into summations over imaginary frequencies using the analytic properties of the dielectric function. This technique is de- 
scribed in detail in Ref. 17. To obtain quasihole selfenergy with wavevector $\mathbf{k}$, we simply put $\omega=\xi_{\mathbf{k}}$ instead of solving the Dyson equation $\omega=\xi_{\mathbf{k}}+\operatorname{Re} \Sigma(\mathbf{k}, \omega)$. This is known 18 to be the correct approximation consistent with the Fermi liquid theory, within the single-loop self-energy approximation of Eq. (11). The quasihole self-energy then becomes a function of wavevector only:

$$
\begin{aligned}
& \Sigma(\mathbf{k})=-\int \frac{d^{3} q}{(2 \pi)^{3}} v_{q} n_{F}\left(\xi_{\mathbf{q}-\mathbf{k}}\right) \\
& -\int \frac{d^{3} q}{(2 \pi)^{3}} v_{q}\left[\frac{1}{\epsilon\left(q, \xi_{\mathbf{q}-\mathbf{k}}-\xi_{\mathbf{k}}\right)}-1\right] \\
& \cdot\left[n_{B}\left(\xi_{\mathbf{q}-\mathbf{k}}-\xi_{\mathbf{k}}\right)+n_{F}\left(\xi_{\mathbf{q}-\mathbf{k}}\right)\right] \\
& -\int \frac{d^{3} q}{(2 \pi)^{3}} T \sum_{\omega_{n}} v_{q}\left[\frac{1}{\epsilon\left(q, i \omega_{n}\right)}-1\right] \\
& \times \frac{1}{i \omega_{n}-\left(\xi_{\mathbf{q}-\mathbf{k}}-\xi_{\mathbf{k}}\right)} \text {. }
\end{aligned}
$$

We directly calculate BGR from Eq. (4) for various hole densities, temperatures and spin polarizations by writing $B G R \equiv|\operatorname{Re} \Sigma(k=0)|$, the magnitude of the real part of band edge self-energy.

\section{RESULTS}

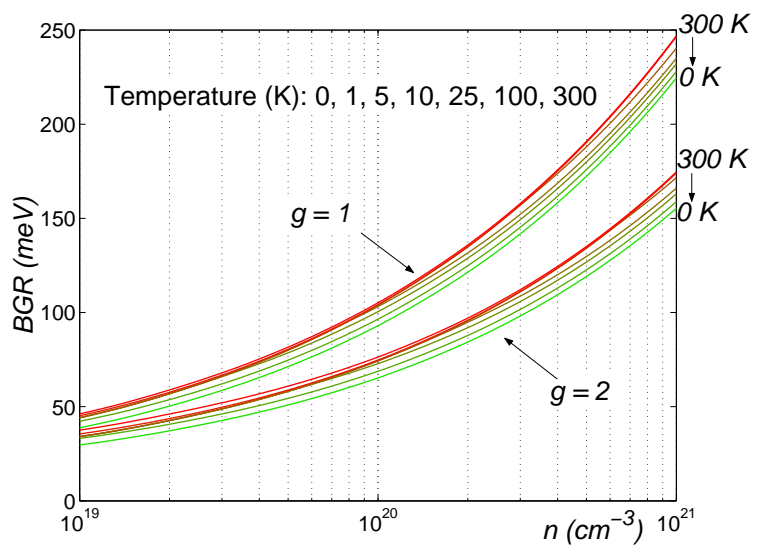

FIG. 2: (Color online.) Calculated band-gap renormalization (narrowing) as a function of hole-density at different temperatures in a $\mathrm{Ga}_{1-x} \mathrm{Mn}_{x}$ As system with spin degeneracy $g=1$ and 2. The upper (lower) 7 curves correspond to $g=1(2)$ case. For each $g$ value, results for 7 different temperatures are shown: $0 K, 1 K, 5 K, 10 K, 25 K, 100 K, 300 K$. Note that BGR results for $T=100 K$ and $300 K$ are almost identical, indicating a saturation temperature effect. $E_{F}=$ $(\mathrm{n} / \mathrm{g})^{2 / 3} \times 249.41 \mathrm{meV}$ where $n$ is in the unit of $10^{20} \mathrm{~cm}^{-3}$.

In Fig. 2]we present the calculated band-gap renormalization as a function of hole-density $n$ at different temperatures. We note from Fig. 2 that the BGR reduces the band gap approximately (but not precisely) as an $n^{1 / 3}$ functional dependence on hole density, and the effect can be as large as a few hundred meVs. Although BGR itself increases in magnitude with hole density, we note that the scaled BGR, i.e. $B G R / E_{F}$, decreases in magnitude with increasing hole density since $E_{F} \sim n^{2 / 3}$. This is consistent with the fact that the relative importance of interaction effects goes down in a quantum Coulomb system with increasing density. Fig. 2 shows that an increasing temperature also enhances BGR, and this enhancement has a tendency to saturate at high temperatures. The increasing BGR with temperature arises from the weakening of screening with increasing temperature. The temperature dependence we obtain is moderate but observable. Even though the percentage correction to BGR due to finite temperature is larger at lower densities, the absolute finite temperature correction is approximately the same for all densities within the range $10^{19}-10^{21} \mathrm{~cm}^{-3}$.

\section{DISCUSSION}

One of the important features of Fig. [2] is that the BGR depends strongly on the magnetic properties of $\mathrm{Ga}_{1-x} \mathrm{Mn}_{x}$ As with the ferromagnetic spin-polarized hole $g=1$ situation having larger, by a factor of 1.5 to 2 BGR, than the corresponding spin-unpolarized $(g=2)$ paramagnetic case. The strong spin degeneracy dependence of BGR apparent in Fig. 2] is understandable on the basis of the fully spin-polarized system having weaker screening since the density of states is lower in magnitude in the polarized system (i.e. $g=1$ is the spin-polarized system versus $g=2$ in the unpolarized system in Eq. (3)). Also, BGR depends indirectly on the Fermi energy, and since the polarized system has a higher $E_{F}$ than the unpolarized state at the same density, the BGR is higher in the fully spin-polarized ferromagnetic system. Such a strong dependence of BGR on the hole spin-polarization (by $50-100 \%$ ) should be reasonably easy to detect experimentally by measuring BGR in $\mathrm{Ga}_{1-x} \mathrm{Mn}_{x}$ As well below and well above the Curie temperature.

We have also carried out calculations for the quasiparticle effective mass and spin susceptibility (or equivalently the Landau $g$-factor) renormalization for the $\mathrm{Ga}_{1-x} \mathrm{Mn}_{x}$ As valence band holes induced by the holehole many-body Coulomb interaction. Our results (not shown) indicate that many-body hole effective mass and spin susceptibility renormalizations are rather small (10$20 \%$ ) at the hole densities of interest in $\mathrm{Ga}_{1-x} \mathrm{Mn}_{x}$ As. This rather small quasiparticle Fermi liquid renormalization in $\mathrm{Ga}_{1-x} \mathrm{Mn}_{x}$ As is consistent with the weakly interacting high-density nature of $\mathrm{Ga}_{1-x} \mathrm{Mn}_{x}$ As. We have also calculated the hole self-energy correction $\Sigma(\mathbf{k})$ in Eq. (4) as a function of wavevector to check for the nonparabolicity introduced by hole-hole interaction. We find that Re $\Sigma\left(k_{F}\right)$ to be slightly $(\sim 10-20 \%)$ larger in magnitude than $\operatorname{Re} \Sigma(k=0)$ at the hole densities of interest, indicating the correlation-induced many-body modification of the hole energy dispersion to be rather small. We 
have also calculated the imaginary part of the hole selfenergy, $\operatorname{Im} \Sigma(k=0)$, to ensure that the quasiparticle picture does not completely break down at the band edge $(k=0)$. We find $|\operatorname{Im} \Sigma(k=0)| / E_{F}$ to be in the 0.2 to 0.05 range for $n=10^{19}-10^{21} \mathrm{~cm}^{-3}$ hole density range of interest, indicating that the quasiparticle band description remains experimentally valid in $\mathrm{Ga}_{1-x} \mathrm{Mn}_{x}$ As in the density range of our interest.

Now we discuss the effect of impurity scattering on the $\mathrm{Ga}_{1-x} \mathrm{Mn}_{x}$ As optical properties, which is likely to complicate the experimental observation of the manybody BGR correction predicted in this paper, particularly since $\mathrm{Ga}_{1-x} \mathrm{Mn}_{x}$ As samples, even in the optimally $(x \sim 0.05)$ doped metallic system, tend to have rather large resistivity indicating strong impurity scattering. Impurity scattering destroys momentum conservation and consequently may strongly affect inter-band optical absorption experiments which depend on wavevector conservation. In particular, strong impurity scattering would lead to two distinct effects on the optical absorption in heavily doped $\mathrm{Ga}_{1-x} \mathrm{Mn}_{x}$ As. First, there will be an upward shift in the valence band edge (i.e. a band gap narrowing) arising from the real part of the self-energy $\Sigma_{\mathrm{i}}$ associated with the hole-impurity interaction. This impurity-induced band-gap narrowing effect has the same practical effect as BGR on the experimentally measured optical absorption gap, and the net band gap narrowing will be a sum of the hole-hole self-energy (i.e. BGR) and the hole-impurity self energy. The holeimpurity self-energy is therefore significant for the optical absorption experiments of interest to us. On the other hand the imaginary part of the hole-impurity self-energy, $\operatorname{Im} \Sigma_{\mathrm{i}}$, leads to a broadening of the momentum eigenstates and is therefore a measure of the level broadening in the optical absorption spectra. This impurity induced level broadening is, therefore, also an important consideration for estimating BGR from the optical absorption spectrum since this will control the broadening of the absorption spectra.

We have obtained the impurity scattering effect on the hole states of $\mathrm{Ga}_{1-x} \mathrm{Mn}_{x}$ As by calculating the holeimpurity self-energy $\Sigma_{\mathrm{i}}(\mathbf{k})$ in the self-consistent singlesite approximation shown in Fig. 1(b). The selfconsistent single-site approximation (Fig. I(b)), which is a substantial improvement on the leading-oder Born approximation, should be qualitatively valid in the metallic regime of $\mathrm{Ga}_{1-x} \mathrm{Mn}_{x}$ As (as long as the carrier are indeed valence band hole states). The integral equation represented by the self-consistent hole-impurity scattering diagrams of Fig. T(b) can be exactly solved for all $\mathbf{k}$ in the strong impurity screening limit (assuming the impurities to be random charge center of density $n_{\mathrm{i}}$ in $\mathrm{Ga}_{1-x} \mathrm{Mn}_{x} \mathrm{As}$ ), and the result for $k=k_{F}$ and $k=0$ are

$$
\begin{aligned}
\Sigma_{\mathrm{i}}(0) & =V_{0} n_{\mathrm{i}}\left(1+\alpha \sqrt{r_{s}}\right)^{-1} \\
\Sigma_{\mathrm{i}}\left(k_{F}\right) & =V_{0} n_{\mathrm{i}}\left[1+\alpha \sqrt{r_{s}} f\left(\beta \sqrt{r_{s}}\right)+i \gamma\right],
\end{aligned}
$$

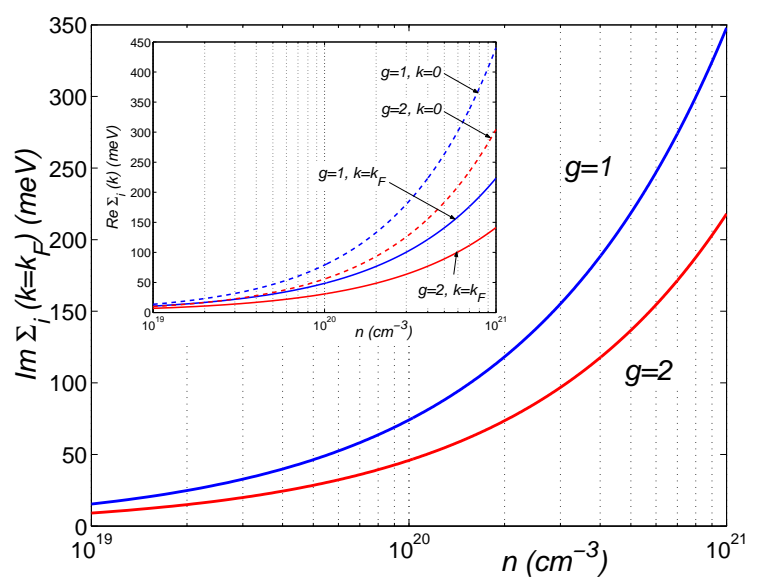

FIG. 3: (Color online.) The contribution of impurity scattering to the real and imaginary quasihole self-energy as a function of hole-density at $k=0, k_{F}$ and $g=1,2$ in a $\mathrm{Ga}_{1-x} \mathrm{Mn}_{x}$ As system. We assume $n_{\mathrm{i}}=n$.

where

$$
f(x)=\frac{1}{2}+\left(\frac{x}{8}-\frac{1}{2 x}\right) \ln \left|\frac{x+2}{x-2}\right|,
$$

and $\alpha=(6 g / \pi)^{1 / 3} ; \beta=2^{2 / 3} 3^{-1 / 3} \pi^{-2 / 3} g^{2 / 3} ; \gamma=\pi / 2$; $r_{s}=(9 \pi / 2 g)\left(a_{B} k_{F}\right)^{-1}$ with $a_{B}$ the effective hole Bohr radius in GaAs; $V_{0}=4 \pi e^{2} / \kappa q_{T F}^{2}$ where $q_{T F}$ is the GaAs hole Thomas-Fermi screening wavevector (and $\kappa$ the GaAs lattice dielectric constant). In Fig. 3 we show our calculated real and imaginary parts of the holeimpurity self-energy assuming an optimal $\mathrm{Ga}_{1-x} \mathrm{Mn}_{x} \mathrm{As}$ metallic system, of $n_{\mathrm{i}} \equiv n$. It is important to point out that our calculated Im $\Sigma$ in Fig. [3 implies a level broadening which is consistent with the measured resistivity of $\mathrm{Ga}_{1-x} \mathrm{Mn}_{x}$ As, providing support for our theoretical approximation. (The actual $\Sigma_{\mathrm{i}}$ may be somewhat smaller than that given in Fig. 3 due to impurity clustering effect ignored in our theory.) We note that the impurity induced level broadening, while being somewhat less in magnitude, is of the same order as the BGR, which may complicate the interpretation of the optical absorption data. (The net band gap shrinkage is given by the sum of BGR (Fig. 2) and the real part of impurity-hole selfenergy given in Fig. 3.) But, we believe that it should still be possible to analyze the optical absorption data to check whether the density dependent optical absorption spectra are consistent with a density, temperature, and spin polarization dependent BGR predicted in our Fig. 2] Especially, the temperature dependence of the impurity scattering effect is very small, and this enables us to identity whether the band shrinking is indeed a many-body BGR effect instead of impurity scattering by examining whether this gap shrinking (if observed) possesses an obvious temperature dependence.

If such a many-body BGR is observed in the experimental data, then that would be strong evidence supporting a valence band RKKY-Zener mechanism for DMS 
ferromagnetism in $\mathrm{Ga}_{1-x} \mathrm{Mn}_{x}$ As. This is because if the holes are located in the impurity band, the BGR effect should be very small because of the large band mass associated with the impurity band. (Note the impurity band BGR effect just mentioned should not be confused with impurity scattering effect mentioned in the last paragraph.) We note that in addition to optical data, STM measurements 19 may also be helpful in the observation of BGR. We emphasize that because of the large defect and impurity content in GaMnAs, invariably present 19 in the low-temperature MBE growth of DMS materials, the observation of BGR will be complicated, but our calculated density, temperature, and magnetization dependence results should enable such a BGR observation of it is present. We also note that at finite temperature electron-phonon interaction ${ }^{20}$ would also contribute to the BGR, but the phonon effect is smaller in magnitude than the exchange-correlation correction in the high hole density GaMnAs of interest to us. Also, the phonon correction does not exhibit a strong density or magnetization dependence.

In summary, we have developed a theory for holehole (and hole-impurity) free carrier interaction induced many-body effects on the optical absorption spectra of $\mathrm{Ga}_{1-x} \mathrm{Mn}_{x} \mathrm{As}$, finding (large) density and magnetization dependent and (moderate) temperature dependent many-body band gap renormalization corrections, which should be observable experimentally provided the holes in $\mathrm{Ga}_{1-x} \mathrm{Mn}_{x}$ As indeed reside in the GaAs valence band, and not in the impurity band.

This work is supported by ONR and ARDA.
1 S. Das Sarma, E. H. Hwang, and A. Kaminski, Solid State Commun. 127, 99 (2003); R. N. Bhatt, M. Berciu, M. P. Kennett and X. Wan, J. of Supercon. 15, 71 (2002); C. Timm, J. Phys. Condens. Mat. 16, R1865 2003; B. Lee, T. Jungwirth, A. H. MacDonald, Sem. Sci. Tech. 17, 393 (2002).

2 A. Chattopadhyay, S. Das Sarma, and A. J. Millis, Phys. Rev. Lett. 87, 227202 (2001).

3 T. Dietl, Semicond. Sci. Technol. 17, 377 (2002).

${ }^{4}$ M. Berciu and R. N. Bhatt, Phys. Rev. Lett. 87, 107203 (2001).

5 T. Dietl, A. Haury, and Y. Merle d'Aubigne, Phys. Rev. B 55, R3347 (1997).

${ }^{6}$ H. Akai, Phys. Rev. Lett. 81, 3002 (1998).

7 S. Sanvito, G. T. G, and N. A. Hill, J. of Supercond. 15, 85 (2002).

8 S. Das Sarma, E. H. Hwang, and A. Kaminski, Phys. Rev. B 67, 155201 (2003).

${ }^{9}$ K. Hirakawa, S. Katsumoto, T. Hayashi, Y. Hashimoto, and Y. Iye, Phys. Rev. B 65, 193312 (2002); E. J. Singley, R. Kawakami, D. D. Awschalom, and D. N. Basov, Phys. Rev. Lett. 89, 097203 (2002).

10 E. H. Hwang, A. J. Millis, and S. D. Sarma, Phys. Rev. B 65, 233206 (2002).

11 S.-R. E. Yang, J. Sinova, T. Jungwirth, Y. P. Shim, and
A. H. MacDonald, Phys. Rev. B 67, 045205 (2003).

12 E. J. Singley, K. S. Burch, R. Kawakami, J. Stephens, D. D. Awschalom, and D. N. Basov, Phys. Rev. B 68, 165204 (2003).

13 S. Das Sarma, R. Jalabert, and S.-R. E. Yang, Phys. Rev. B 41, 8288 (1990).

${ }^{14}$ G. Tränkle, H. Leier, A. Forchel, H. Haug, C. Ell, and G. Weimann, Phys. Rev. Lett. 58, 419 (1987); G. Tränkle, E. Lach, A. Forchel, F. Scholz, C. Ell, H. Haug, G. Weimann, G. Griffiths, H. Kroemer, and S. Subbanna, Phys. Rev. B 36, 6712 (1987); G. Bongiovanni and J. L. Staehli, Phys. Rev. B 39, 8359 (1989).

15 L. Hedin, Phys. Rev. 139, A796 (1965).

16 M. L. Tiago, S. Ismail-Beigi, and S. G. Louie, Phys. Rev. B 69, 125212 (2004).

17 S. D. Sarma, V. M. Galitski, and Y. Zhang, Phys. Rev. B 69, 125334 (2004)); Y. Zhang and S. Das Sarma, Phys. Rev. B 70, 035104 (2004).

18 Y. Zhang and S. Das Sarma, Phys. Rev. B 71, 045322 (2005); T. M. Rice, Ann. Phys. (N. Y.) 31, 100 (1965).

19 R. M. Feenstra, J. M. Woodall, and G. D. Pettit, Phys. Rev. Lett. 71, 1176 (1993); C. Timm, F. Schäer, and F. von Oppen, Phys. Rev. Lett. 89, 137201 (2002).

20 M. Cardona, Sol. St. Commun. 133, 3 (2005). 